\title{
Currículo escolar, conocimiento [matemático] y prácticas sociales: posibilidades otras en una comunidad indígena Gunadule'
}

Carolina Tamayo-Osorio"

\section{Resumen}

Este artículo es resultado de una investigación de maestría titulada: (re)significación del currículo escolar indígena, relativo al conocimiento [matemático], desde y para las prácticas sociales: el caso de los maestros de la comunidad Dule en Alto Caimán (TAMAYO-OSORIO, 2012). En coherencia el objetivo de investigación fue: (re)significar el currículo escolar indígena, relativo al conocimiento [matemático], desde y para las prácticas sociales de la comunidad Dule en Alto Caimán. El horizonte teórico se orientó desde una perspectiva sociocultural de la educación matemática considerando la Etnomatemática y la interculturalidad. Este estudio es de orden cualitativo con visión crítico-dialéctica, basado en una investigación colaborativa. Podemos afirmar que hablar de currículo escolar indígena, relativo al conocimiento [matemático], desde una perspectiva crítica implica (re)conceptualizaciones, donde se hagan visibles no sólo las concepciones teóricas, políticas y culturales que sustentan la sociedad que se quiere construir, sino que también se manifieste la dialéctica entre prácticas sociales y conocimientos [matemáticos].

\section{Palabras-clave}

Educación indígena - Currículo crítico - Etnomatemática Educación matemática - Prácticas sociales.

I- Esta investigación fue orientada por la Prof. Dra. Diana Jaramillo Quicéno, agradecemos sus contribuciones en todo el proceso académico que hoy nos conduce a la publicación de este artículo. Agradecimientos, al Comité para el Desarrollo de la Investigación de la Universidad de Antioquia (CODI) por la financiación de esta investigación que fue parte del proyecto intitulado Prácticas sociales, currículo y conocimiento matemático, además a los hermanos y hermanas Gunadule por sus voces y contribuciones en este trabajo.

II- Universidade Estadual de Campinas, Brasil. Contacto: carolina.tamayo36@gmail.com 


\title{
School curriculum, [mathematical] knowledge and social practices: other possibilities in an indigenous Gunadule community
}

Carolina Tamayo-Osorio"

\begin{abstract}
This article is the result of a study entitled (Re)interpretation of indigenous school curriculums in terms of [mathematics] knowledge from and for social practice: the case of Dule teachers from Alto Caimán. Our research project objective was to (re)signify the indigenous school curriculum in terms of [mathematics] knowledge from and for social practices in the Dule community of Alto Caimán. This qualitative study conducted from the socio-cultural perspective of mathematics education, with contributions of ethnomathematics and from interculturalism, and from a criticaldialectical perspective, was based on a collaborative investigation. From the results obtained in this study we learned that discussing indigenous school curriculums in terms of [mathematics] knowledge implies (re)conceptualizations, from which not only theoretical, political and cultural conceptions that underpin the type of society that one desires to build, but also the dialectic between social practices and [mathematics] knowledge are evidenced.
\end{abstract}

\section{Keywords}

Indigenous education - Critical curriculum - Ethnomathematics Mathematics education - Social practices.

\footnotetext{
I- This research was supervised by Professor Diana Jaramillo Quiceno, whom we thank for her contributions throughout the academic process that has led to the publication of this jornal paper. We also thank the Comité para el Desarrollo de la Investigación (CODI - Committee for Research Development) of Universidad de Antioquia for funding the study. This research was part of the project entitled Social practices, curriculum and Mathematics knowledge. Finally, we wish to thank Gunadule women and men for their voices and contributions to this work.

II- Universidade Estadual de Campinas, Brasil. Contact: carolina.tamayo36@gmail.com
} 


\section{Introducción}

Pensar sobre la educación indígena $y$, en particular, sobre la construcción de currículos que atiendan la diversidad y las diferencias de los diversos grupos culturales en Colombia, ha sido desde hace algunos años objeto de estudio del grupo de investigación Matemática, Educación y Sociedad-MES de la Universidad de Antioquia (Medellín, Colombia), estudios estos, que nos han posibilitado de manera colectiva, algunas ideas, reflexiones $\mathrm{y}$ preocupaciones relativas a la educación en general, y específicamente la educación matemática. Algunas de esas reflexiones conjuntas nos han hecho pensar sobre la necesidad de problematizar los posibles diálogos entre las prácticas sociales de la comunidad Gunadule de Alto Caimán ${ }^{2}$ (poseedora de una sabiduría) y los conocimientos de otras culturas que llegan a la comunidad por medio de la escuela del Estado ${ }^{3}$.

En este artículo presentamos algunos de los hallazgos de la investigación desarrollada por Tamayo-Osorio (2012) que ya ha sido discutida en diversos espacios académicos y que corresponde a las categorías de análisis. Optamos por orientar nuestras reflexiones y análisis hacia una escuela indígena Dule que pueda atender las necesidades de la comunidad en la que está inmersa y que ayude a fortalecer y preservar la identidad cultural. El objeto de estudio realizado fue la (re)significación del currículo escolar indígena relativo al conocimiento [matemático] desde y para las prácticas sociales de la Comunidad indígena

1- Investigaciones como Berrío (2009), Higuita (2011), Green Stócel (2007; 2011), Santacruz y Castaño (2012).

2- El pueblo Dule tiene su territorio en el golfo de Urabá y en la región del Darién, específicamente en Necoclí (Antioquia, Colombia), este territorio se encuentra dividido en tres zonas: Bajo, Medio y Alto Caimán. Esta investigación se desarrolló de forma colaborativa desde y para la comunidad de Alto Caimán en el Centro Educativo Rural Alto Caimán.

3- Cuando usamos el término escuela del Estado nos estamos refiriendo al lugar, al espacio y a la idiosincracia que caracterizan la escuela en una perspectiva occidental, los maestros usan esta terminología para referirse a dicho espacio, por tanto la adoptamos como producto de nuestro trabajo de campo.
Gunadule de Alto Caimán, basados en diálogos interculturales con la Casa del Congreso ${ }^{4}$.

Asumimos la (re)significación como un elemento para dar cuenta de que los procesos de significación cultural e individual que siempre están en constante movimiento, los sujetos van enriqueciendo el significado de sus conocimientos en el marco de los grupos humanos (BAJTÍN, 2009). Todos fuimos unos con otros significando y (re)significando, pues en las reflexiones, en los diálogos y en cada una de las etapas del desarrollo de esta investigación. Nos acompañaron en este camino los planteamientos teóricos y metodológicos de D’Ambrosio (1994; 1999; 2008), Monteiro (2005), Monteiro, Sena y Santos (2007), Monteiro y Mendes (2011), Jaramillo (2011), Moura et al. (2010), Moura (2011), Bajtín (1986; 2009), Silva $(1995 ; 1998 ; 1999 ; 2010)$, Scandiuzzi (2009), entre otros.

\section{Tejiendo colaborativamente:}

orientaciones metodológicas

Comenzamos a inquietarnos por desarrollar una investigación que problematizara y cuestionara el currículo escolar indígena Dule, enfocados en el conocimiento [matemático] ${ }^{5}$, pues había un interés de la comunidad Gunadule, expresado por medio de los profesores indígenas de dicha comunidad que eran estudiantes de la Licenciatura en Pedagogía de Madre Tierra $^{6}$ de la Universidad de Antioquia, de la Organización Indígena de Antioquia (OIA) ${ }^{7}$,

4- La Casa del Congreso de la comunidad Dule es un lugar sagrado, donde tanto niños, como jóvenes y adultos se reúnen para escuchar a los caciques (sailas) que en lengua Dule son llamados saglamala. En ese espacio, los saglamala socializan, desde la oralidad, el saber familiar, el saber cultural y las tradiciones a los demás miembros de la comunidad.

5- En vista de lo problemática que se tornó la discusión acerca de los hallazgos encontrados, optamos (tras innúmeras discusiones con los profesores) por asumir esa connotación, que ganará sentido a lo largo del texto.

6- La Universidad de Antioquia, mediante la firma del Convenio Marco de Cooperación no 019 de 2004 con la Organización Indígena de Antioquia, dio paso a la creación del Programa de Educación Indígena, adscrito a la Facultad de Educación (Resolución Académica 1752 del 18 de agosto de 2005).

7- La OIA es "una organización social de base, constituida jurídicamente como entidad de derecho público, encargada de la representación política de las comunidades indígenas de Antioquia pertenecientes los pueblos Dule, Senú y Embera" (OIA, 2007, p. 6). 
del Instituto Departamental para la Educación Indigena (INDEI) ${ }^{8}$ y de los investigadores de la Universidad de Antioquia. De esa forma el camino que seguimos se construyó a partir de las creencias, de los intereses, de los conocimientos, de la sabiduría de las personas que se unieron a esta idea de pensar una educación otra para la comunidad de Alto Caimán. Hubo diálogos, desde las diferentes formas de ser y de hacer de los participantes, con el objetivo común de buscar alternativas posibles en el campo educativo indígena.

Es importante esclarecer, que asumimos el desde y para, en primer lugar, porque en el desarrollo de la investigación estuvimos mirando las prácticas sociales como unidad dialéctica con los conocimientos [matemático] para pensar elementos otros para una educación matemática indígena. Y, en segundo lugar, porque consideramos que esta investigación debería estar enfocada hacia la comunidad y reconocida por la misma, a partir de la elaboración de un trabajo de campo que posibilitara poner en diálogo a los expertos de las prácticas sociales, los saglamala ${ }^{9}$ de la comunidad, los maestros indígenas y los investigadores de la universidad. Este último aspecto fue reconocido por el maestro indígena Richard Nixon Cuéllar al plantear que,

[...] la investigación que hemos ${ }^{10}$ venido desarrollando ha sido muy importante porque empezamos [maestros e investigadores] a trabajar con los sailas; los llamamos y ellos compartieron sus conocimientos. Este proyecto se hace una realidad [se legitima para la comunidad] porque cuenta con el apoyo de los sailas, que reconocen los hallazgos y las

8- El Instituto Departamental para la Educación Indígena - INDEI, es una asociación civil sin fines de lucro que fue creada en el año 1999 y posee la Personería Jurídica № 1.669.955 por resolución de la Inspección General de Justicia № 774/00. El INDEl, se crea con el propósito principal de "Estudiar la Condición Humana y contribuir a mejorar su desarrollo" (INDEl, 2011, p. 1).

9- El saila o sagla es la máxima autoridad de la comunidad Dule; es un sabio y un líder de la comunidad. Saglamala es plural de la palabra.

10- La cursiva es nuestra. propuestas. Si estuviéramos solos, de pronto la comunidad nos rechazaría la propuesta, pero creo que ahora no, pues tenemos el apoyo del cacique y de los sabios. (Fragmento de entrevista concedida para esta investigación por Richard Nixon Cuéllar el 28 de noviembre de 2011).

Las palabras del maestro hacen referencia al diálogo establecido entre todos los que mirábamos el mismo objeto de investigación, pero desde lugares diferenciados; se manifiesta una relación entre necesidades de la escuela, las apuestas políticas que se lideraban en la comunidad Dule de Alto Caimán y las apuestas lideradas desde la Universidad de Antioquia con los diferentes investigadores; además, vimos la necesidad y la importancia que tenía investigar colaborativamente para conseguir tejer y destejer la problemática y las alternativas concretas frente al proceso de (re)significación del currículo escolar, relativo al conocimiento [matemático], que venía siendo desarrollado desde el pueblo Dule. Nos encontramos, así, en el transcurso de la investigación asumiendo el reto de investigar colaborativamente, problematizando el currículo escolar indígena, relativo al conocimiento [matemático], cada uno desde un lugar diferente, pero todos con el mismo horizonte; todos dispuestos a dar y a recibir para construir alternativas concretas y reflexiones más profundas frente al contexto sociopolítico del pueblo Dule en el que se desenvuelve la discusión frente al currículo escolar indígena. Vivenciamos como lo plantean Boavida y Ponte que,

Toda colaboración es un proceso emergente, marcado por la imprevisibilidad y lleno de negociaciones y decisiones (Grey, citado por Stewart, 1997). En este proceso, es fundamental que los participantes manifiesten apertura sobre la forma en que se relacionan unos con otros, disponiéndose a un continuo dar y recibir, asumiendo una responsabilidad 
conjunta por la orientación del trabajo y siendo capaces de construir soluciones para los problemas basados en el respeto por las diferencias y las particularidades individuales. En un trabajo de colaboración existe, necesariamente, una base común entre los distintos participantes, que tiene que ver con los objetivos y las formas de trabajo y de relación. (BOAVIDA; PONTE, 2011, p. 129).

En este sentido, los mismos autores reconocen que "en realidad, la colaboración constituye una estrategia fundamental para lidiar con problemas que parecen demasiado pesados para ser enfrentados en términos puramente individuales" (p. 126). En nuestro caso, la colaboración entendida en este sentido, como la posibilidad de trabajar conjuntamente por un objetivo en común. De esa forma, esta investigación emergió y se constituyó como investigación colaborativa caracterizada por una mirada cualitativa con un enfoque críticodialéctico (SÁNCHEZ, 1998); en ella concebimos los sujetos como el resultado de los procesos sociales, como transformadores de su medio y constructores de la historia. Este enfoque críticodialéctico cuestiona la visión estática de la realidad, lo que posibilita ver el carácter dinámico e histórico de los fenómenos educativos. Desde esta perspectiva, se dirigen, la teoría y la práctica, hacia un pensar y actuar en un proceso cognitivo y transformador de la naturaleza; además, proponen presentar los conflictos e intereses de las situaciones o fenómenos.

El trabajo de campo se constituyó con dos visitas in-situ de los investigadores a la comunidad y dos visitas en la Universidad. En el diálogo de todas las voces de esta investigación emergieron cuatro categorías de análisis; para efecto de este artículo, y considerando los aportes que podemos ofrecer al campo de la Educación Matemática y especialmente de la Educación en general, presentamos dos categorias el currículo escolar indigena, relativo al conocimiento [matemático]: tensiones, sentidos e identidad y el maestro indígena Dule en situación de frontera, finalmente presentaremos algunas consideraciones.

\section{El currículo escolar indígena, relativo al conocimiento [matemático]: tensiones, sentidos e identidad}

La educación indígena del pueblo Dule en Colombia se ha discutido con mayor intensidad en los últimos 20 años, pues esta comunidad indígena ha venido participando de forma activa en el proceso de construcción de los marcos legales que regulan la educación indígena en el país, y con mayor fuerza, a partir de la reforma hecha a la constitución en 1991, en la que se reconoce a las comunidades indígenas -a partir de su organización social, costumbres, lenguas, creencias y tradiciones-. En este sentido, el Ministerio de Educación Nacional -MEN-, en la Ley 115 de 1994, a partir del programa de Etnoeducación ${ }^{11}$ ha buscado implementar a nivel nacional el modelo de escuela nueva ${ }^{12}$ para comunidades afrodescendientes, campesinas e indígenas.

En la propuesta de escuela nueva, como lo señala el MEN (COLOMBIA, 2011a), se plantea el componente curricular y pedagógico desde aspectos metodológicos en el interior del aula y como facilitador de la articulación de las áreas obligatorias y fundamentales ${ }^{13}$. Esta visión da

11- La Etnoeducación es entendida en el artículo 55 de la Ley general de Educación 115 de 1994, "como la educación para grupos étnicos, la que se ofrece a grupos o comunidades que integran la nacionalidad y que poseen una cultura, una lengua, unas tradiciones y unos fueros propios y autóctonos". (COLOMBIA, 1994, p. 19).

12- El modelo curricular de escuela nueva, como lo señala MEN (COLOMBIA, 2011a), que surgió en el país en la década de los años 70, como una propuesta para las necesidades educativas de los niños de primaria de las zonas rurales del país.

13- Estas áreas son descritas en MEN (COLOMBIA, 1994) en el apartado de áreas obligatorias y fundamentales, en el capítulo 1, sección tercera, artículo 23. Los grupos de áreas obligatorias y fundamentales que comprenderán un mínimo del $80 \%$ del plan de estudios, son los siguientes: 1. Ciencias naturales y educación ambiental. 2. Ciencias sociales, historia, geografía, constitución política y democracia. 3. Educación Artística. 4. Educación ética y en valores humanos. 5. Educación física, recreación y deportes. 6. Educación religiosa. 7. Humanidades, lengua castellana e idiomas extranjeros. 8. Matemáticas. 9. Tecnología e informática. 
indicios sobre la poca correspondencia de dicho modelo educativo con los contextos de estas comunidades, que adicionalmente entre ellas son diferentes -no solo en prácticas sociales, sino fundamentalmente en cosmogonías $\mathrm{y}$ cosmovisiones-.

En la búsqueda de interrelaciones en condiciones interculturales ${ }^{14}$ entre las propuestas gubernamentales y los deseos e intereses del pueblo Gunadule Colombiano, se han desarrollado diversos proyectos de investigación en torno al currículo escolar indígena Dule y la escuela del Estado como institución no indígena, pero que debe ser apropiada y (re)pensada por este pueblo: Green Stócel, Cardozo, y Ochoa (1995); en el 2010 -el proceso que iniciaron en esta investigación comienza a tornarse centro de discusión en el Convenio MEN-Ibgigundiwala-; Green Stócel (2007; 2011) y Santacruz y Castaño (2012).

En la voz de Nazario Uribe como líder político de la comunidad, podemos ver la importancia que ha tenido para el pueblo Dule la discusión frente al currículo escolar indígena en relación con su plan de pervivencia cultural y su plan de salvaguarda ${ }^{15}$ en relación intercultural con otros pueblos,

Soy un convencido de que hay que luchar por el currículo propio, es una lucha, un proceso y hay que concientizar muy bien a la comunidad y explicarle muy bien los términos...porque confundimos la escuela con la educación...son términos que tenemos explicar para que sean más adecuados y que la gente entienda. (Fragmento de entrevista concedida para esta investigación por Nazario Uribe el 29 de octubre de 2011).

Vemos, en primer lugar, la existencia de una preocupación por unas políticas educativas

14- En el sentido de Walsh (2008).

15- Bajo la sentencia T-025 de 2004 se explicita en el Auto 004/09 expedidos por la Corte Constitucional busca que el Estado Colombiano proteja a 34 pueblos indígenas que están en riesgo de exterminio por desplazamiento, Los elementos centrales de esta sentencia están contemplados según la Corte Constitucional (2009). que desconocen los procesos históricos y culturales de las comunidades y que privilegian a unos sobre otros; en segundo lugar, por la formación de maestros que trabajan en estos contextos; en tercerlugar, por un currículo escolar que se propone y cómo se propone. Esta última preocupación hace referencia especialmente a la disciplinarización y fragmentación ${ }^{16}$ de los conocimientos que son enseñados a niños y a jóvenes en sus comunidades, desde una institución llamada escuela. Vemos que las discusiones sobre el currículo se han limitado a la idea de cómo organizarlo o qué contenidos enseñar, desconociendo otros elementos que son constitutivos del mismo (SILVA, 2010). En esta visión del currículo, la función de la escuela y del docente, está dada bajo la idea de ejecutores de lo que se debe enseñar, significa seleccionar y organizar los objetivos, contenidos $\mathrm{y}$ estrategias formativas que encuadran a los sujetos en formación, con la finalidad de que esos estudiantes respondan positivamente a las pruebas estatales.

En ese sentido, Ferri afirma que la "definición de cuales contenidos son válidos y/o significativos en cada contexto escolar va depender de la compresión de cuáles conocimientos deben ser apropiados como productos de la cultura y como estas experiencias seguirán construyendo nuevos conocimientos" (2002, p. 88). Ferri señala, citando a Sacristán (1998), que los contenidos encaminados desde las reflexiones comunitarias en las comunidades indígenas, muestran "que los nuevos significados de los contenidos, no se encajan con facilidad en las áreas o disciplinas en las cuales tradicionalmente se ha distribuido el currículo [...]” (p. 88). Al respecto, Monteiro y Mendes hacen un llamado al reconocer que,

[...] la valorización y la legitimación de prácticas y saberes excluidos del contexto

16- Este término lo comprendemos aquí, como la fragmentación dada al conocimiento en disciplinas: matemáticas, lengua castellana, ciencias naturales, religión, etc., pero también a la fragmentación de los cuerpos, de los tiempos y los espacios. 
escolar procuran, ante todo, permitir a los sujetos, no sólo su identificación con el ambiente escolar, entendiendo éste como un espacio por ellos comprendido, sino también su participación en debates que promuevan la interacción y la clarificación de las relaciones de poder que sustentan los procesos de legitimación de producción de saberes en las diferentes prácticas. (MONTEIRO; MENDES, 2011, p. 42).

Coincidimos con este llamado a integrar en las dinámicas escolares los conocimientos oriundos de las prácticas sociales, que no necesariamente son adjetivados en todas las culturas usando las mismas palabras. Ello nos explicitó la importancia de (re)pensar y discutir conjuntamente el currículo escolar propuesto para el pueblo Dule en función de una dialéctica entre conocimientos y prácticas sociales de forma indisciplinar ${ }^{17}$. Comprendiendo que,

[...] práctica social es toda acción o conjunto intencional y organizado de acciones físico-afectivas-intelectuales realizadas, en un tiempo y espacio determinados, por un conjunto de individuos, sobre el mundo material y/o humano y/o institucional y/o cultural, acciones estas que, por ser, siempre, y en cierta medida, y por un cierto período de tiempo, valorizadas por determinados segmentos sociales, adquieren una cierta estabilidad y se realizan con cierta regularidad. (MIGUEL; MIORIN, 2004, p. 165).

Así, asumimos en este proyecto que las prácticas sociales están en dialéctica con el conocimiento producido en la actividad humana, pues cuando se modifica la realidad externa al sujeto, al mismo tiempo se transforman diversas cualidades de los conocimientos que ya se tenían apropiados. En palabras de Lenin "la dialéctica puede ser concisamente definida

17- En el sentido de Miguel (2010). como la doctrina de la unidad de opuestos" (LENIN, 1977 apud DAVIDOV, 1988, p. 17); es decir, mientras que desde las prácticas sociales el hombre se ve enfrentado a nuevas situaciones, los conocimientos que inicialmente se asociaban a dicha actividad humana simultáneamente se ven modificados, pues no son suficientes para el desarrollo de la actividad a ser ejecutada.

Es posible identificar en la voz del profesor Francisco Martínez cómo las prácticas sociales y el conocimiento se tornan unidad y como desde dicha interacción dialéctica hombre-naturaleza se posibilitan más elementos en nuestro caminar sobre la (re)significar el concepto de currículo escolar indígena relativo al conocimiento [matemático] desde una perspectiva indisciplinar,

En la escuela del Estado, en el salón, uno hace teorías...En cambio en la práctica social uno trabaja es practicando, uno da más conocimientos, ya uno ve el camino de lo que quiere hacer. Uno calcula o también puede dividir. Ahí salen muchas cosas... El cultivo del plátano no solo sirve para saber matemáticas sino también para alimentar y para la medicina. (Fragmento de trabajo de discusión desarrollado para esta investigación el 27 de julio de 2011).

Las palabras del profesor hacen referencia a que las prácticas sociales en las que se movilizan los conocimientos [matemáticos] en la comunidad, deben ser comprendidas desde la sabiduría indígena de este pueblo caracterizada por una fuerte relación con la Madre Tierra que se refleja en su cosmogonía y cosmovisión, de la cual los conocimientos eurocéntricos que llegan a la comunidad, por medio de la escuela, carecen. Se pone de manifiesto, que los sentidos y significados que son producidos en las prácticas culturales en ambas culturas son legitimados ${ }^{18}$ y valorizados de formas diferentes. Por ejemplo,

18- El término legitimación será asumido aquí de acuerdo con Monteiro Sena y Santos como "la aceptación y validación de los conceptos y valores 
En la cultura Dule los números 4, 8 y 12 están cargados de simbolismos: el hombre en el universo se encuentra en el cuarto nivel; son cuatro los días del encierro de la niña en la primera menstruación ${ }^{19}$; son cuatro los troncos para el fuego que son imprescindibles para el casamiento; son cuatro días de acercamiento que debe darse en los recién casados, antes de la unión real; son cuatro las etapas de prueba que ha pasado la Madre Tierra y cuatro los nombres que ha recibido el continente de Abya Yala: Gwalagun Yala, Dagargun Yala, Yaladingua Yala, Abya Yala; ocho los hermanos que lucharon contra el mal; Ibeorgun enseña doce tipos de gammdur igar (flautas); según el Sagla Igwanabiginya, son doce los grandes nergan que bajaron después de Orgun. (WAGUA; GREEN STÓCEL, 2005 apud GREEN STÓCEL, 2011, p. 167).

La Madre Tierra se torna centro de la cosmogonía y cosmovisión del pueblo Dule; de esa forma guía y da sentido a los conocimientos que han sido producto de las interacciones entre hombre y Madre Tierra o Nabgwana ${ }^{20}$. De este modo, pensar otra escuela y otro currículo escolar indígena relativo al conocimiento [matemático] para la comunidad Dule supone considerar el espacio escolar como un lugar de diálogo y de debate, un lugar de encuentro entre culturas con conocimientos [matemáticos] diferenciados, un lugar donde se dé cabida a las subjetividades de los alumnos. Una escuela que se configure como un espacio de diálogo entre culturas, en la que no se sobrepongan unos conocimientos sobre otros, que sea posible comprender, atender y asumir la diversidad cultural.

por los grupos que los utilizan. Está legitimación no es única y universal, no es una verdad abstracta y totalitaria, por el contrario, ella representa una relación dialéctica de opresión y resistencia, dependiendo de la forma con que se hace presente en el grupo..." (2007, p. 52).

19- Tanto para el encerramiento de la niña como en el casamiento, los días son ocho para las comunidades Gunadule en Arquía (Chocó) y Caimán Nuevo (Urabá) en Colombia.

20- Nombre que se da a la Madre Tierra en Dule, madre de la abundancia.
En este sentido, es necesario comprender, como lo plantea Silva (2010) y como lo asumimos nosotros, que hablar de currículo escolar no es apenas preguntarnos y respondernos por el qué enseñar. En esta perspectiva crítica, el autor plantea que a la hora de pensarnos el currículo, nos corresponde, también, indagar respecto al ¿por qué son pertinentes unos conocimientos y no otros?, ¿cómo enseñar?, ¿para qué enseñar?, y, fundamentalmente, ¿a quién se enseña? Estas preguntas implican negociaciones y discusiones de orden epistemológico, pero sobre todo de orden ontológico, pues el currículo escolar es un asunto de identidad. Además, para este autor,

[...] Un currículo desde la perspectiva crítica no puede pasar de largo sobre las preocupaciones y vivencias centrales de los niños y jóvenes de este tiempo, [...], es tornarlo relevante para la vida social de esta convulsionada época. Evaluar en qué medida el currículo está implicado con estas cuestiones puede ser una forma de reconocer su carácter crítico y descolonizador. (SILVA, 1998, p. 8).

Estos planteamientos nos ayudan a centrar las discusiones sobre el currículo en la pregunta ¿a quién enseño? Y no en la pregunta ¿qué enseño? En ese sentido, se abre la posibilidad de reconocer a los sujetos desde el lugar que ocupan en el mundo. Sujetos que hacen parte de ciertas prácticas sociales; sujetos con intereses y visiones diversas; sujetos con diferentes maneras de comprender la realidad. Es decir, en la perspectiva crítica del currículo, en la cual apostamos, se busca la recuperación de un sujeto olvidado. Un sujeto pensado desde los productos evaluables y medibles (en su hacer y en su saber), apenas, derivados de sus conocimientos impartidos en las escuelas del Estado.

La propuesta de pensar un currículo crítico, implica además “[...] entender el saber/ hacer matemático a lo largo de la historia de la humanidad, contextualizado en diferentes grupos de interés, comunidades, pueblos $\mathrm{y}$ 
naciones" (D’AMBROSIO, 2008, p. 17). Esto significa algo más que hacer un reconocimiento del saber/ hacer matemático en los pueblos, se trata de entender lo que en estos pueblos se produce como conocimientos [matemáticos] en relación con los desarrollos históricos, sociales, políticos, económicos y culturales propios de un grupo humano, como lo plantea el programa de investigación de Etnomatemática. Lo que significa, que es necesario no comparar los cocimientos de las comunidades indígenas con el conocimiento en el cual hemos sido formados -el de la cultura occidental. De lo que se trata es de entender el saber/hacer producido allí, en cada pueblo, desde sus dinámicas, desde sus lógicas. La validación de éste conocimiento solo tiene sentido en la cultura en la que se desarrolla, vimos que en esta comunidad hay tensiones referidas a la compresión del término matemáticas, esas matemáticas disciplinares que aparecen el currículo escolar desconectado da cotidianidad. Existen diferentes sentidos producidos hacia ese término, tanto desde las comunidades académicas occidentales como desde la comunidad Dule. Por ejemplo, en las raíces lingüísticas ancestrales del pueblo Dule no existe la palabra matemáticas.

A raíz de la dicotomía que se ha generado en los currículos escolares entre los saberes escolares relativos al conocimiento [matemático] y los saberes cotidianos, oriundos de las prácticas sociales, se ha generado un tipo exclusión provocada por el conocimiento (KNIJNIK, 1997), dicha dicotomía, dicha exclusión, está centrada en la ausencia de los conocimientos [matemáticos] de las prácticas sociales de los alumnos en el currículo escolar.

En palabras de Monteiro (2005) cuando los saberes escolares desconocen o deslegitiman otra forma de conocimientos y de saberes, se genera un modo de exclusión social, pues esto conlleva a la deslegitimación de las prácticas sociales que dan sustento a dichos saberes. Estos planteamientos hacen referencia a que una de las formas de exclusión producida en el currículo escolar se da cuando este legitima determinados conocimientos y silencia otros, como es el caso de la comunidad Dule de Alto Caimán. En este sentido coincidimos con Silva cuando afirma que,

El currículo de la escuela está basado en la cultura dominante: él se expresa en un lenguaje dominante, él es transmitido a través del código cultural dominante. Los niños de las clases dominantes pueden fácilmente comprender ese código, pues durante toda su vida en ellos estuvieron todo el tiempo inmersos, en esos códigos, [...]. En contraste, para los niños y los jóvenes de las clases dominadas, ese código es simplemente indescifrable. Ellos no saben de lo que se trata [...]. El resultado es que los niños y jóvenes de las clases dominadas solo pueden enfrentar el fracaso, quedándose en el camino (SILVA, 2010, p. 35).

Otro aspecto que podemos analizar, es que desde la escuela Estado se piensa la formación de sujetos en relación con unos conocimientos [matemáticos] que han sido configurados desde la cultura occidental -o dominante-. En ese sentido, el currículo escolar indígena busca reflejar la idea de que en la escuela del Estado se relativicen las normas que rigen el calendario y el ritmo de las actividades escolares, para que de esa forma los niños y maestros indígenas puedan asistir a las actividades de las escuelas ancestrales de forma paralela.

Las reflexiones teóricas que hasta ahora hemos expuesto, y la lectura desde el diálogo con la comunidad Dule nos posibilitaron el cuestionamiento a lo que hoy llamamos -desde occidente- de conocimiento [matemático]. Para la comunidad Dule, el conocimiento [matemático] no existe, en la lectura que occidente lo comprende. Para la cultura Dule existe el conocimiento. Es por ello que en este trabajo hemos optado por hablar del conocimiento [matemático]. En palabras de los sailas Jaime Melendres y Faustino Vicente Arteaga, 
Nuestros conocimientos son ancestrales, porque cada pueblo de acuerdo a su origen tiene su historia de donde originó $^{21}$. Porque cada cultura tiene su mundo de conocimientos, su filosofía y por la evolución del tiempo también va cambiando su modo de pensar; pero se sigue conservando lo que uno es. En nuestro caso se sigue siendo Dule. (Fragmento de trabajo de discusión desarrollado para esta investigación el 20 de abril de 2012).

En esa misma dirección, por ejemplo, Scandiuzzi (2009) desde un estudio realizado en el Parque Nacional del Xingu en Brasil con el pueblo indígena de Kuikuro, plantea que cuando se trata de educación indígena,

[...] creemos que hablar de matemática (producida por la cultura blanca escolar) para ese grupo suena como un mensaje de afuera. Para los más sensibilizados con la historia de su pueblo, es hablar de conquista, es hablar de algo construido por el dominador, que se sirve de esos instrumentos para ejercer su dominio. Estamos procurando mezclar agua y aceite: matemática e indígena. Es evidente que la mistura se logra. [...] todo será hecho para satisfacer el cumplimiento de un programa, de requisitos mínimos para que el indígena obtenga algunos créditos y acumule credenciales, para salir de la condición natural de incapaz. (SCANDIUZZI, 2009, p. 18).

Hacer parte de una cultura es apropiarse de un cuerpo de conocimientos, generados desde, para y en las prácticas sociales, que posibilitan interactuar con los sujetos de manera individual y colectiva. Así, en esa interacción se construyen, legitiman y validan conocimientos para construir nuevos conocimientos e instrumentos que permitan al hombre vivir mejor, que posibiliten el desarrollo de las prácticas sociales

21- La cursiva es nuestra. de forma más rápida. Puesto que las necesidades del hombre tienen tanto un carácter individual como colectivo y los conocimientos asociados a ellas se transforman al igual que las prácticas sociales. En palabras del profesor Richard Nixon Cuellar: "ahora entendi que las prácticas sociales no solamente son cultivos, sino que es todo lo que hacemos en la comunidad ${ }^{22}$ : reuniones, cantos de arrullo, tejer molas, chaquiras, elaborar canastos" (Fragmento de trabajo de discusión desarrollado para esta investigación el 28 de julio de 2011) y allí es justamente donde se movilizan conocimientos que permiten que dichas prácticas se desarrollen de forma inequívoca.

Esta postura nos lleva a reconocer que el conocimiento [matemático] Dule tiene sentido en la medida en que se reconocen las raíces culturales e históricas, y en la medida en que dicho conocimiento esté relacionado con la búsqueda de respuestas a problemas que se presentan en las necesidades originarias de las prácticas. Para el pueblo Dule las matemáticas, como todo tipo de conocimiento, hacen parte de cada una de sus prácticas sociales, de sus escuelas ancestrales; el conocimiento milenario no es fragmentado y hace parte de los significados de vida ${ }^{23}$.

Se han venido construyendo propuestas que consideran como principios fundamentales la cosmogonía Dule como MEN-Ibgigundiwala (2011b), en la que el congreso Gunadule de Colombia ha tejido una propuesta para pensar la educación de jóvenes, niños y adultos en la búsqueda de conservación de la identidad Dule, en las palabras Richard Nixon Cuéllar,

[...] nosotros los pueblos indígenas [como el Dule] estamos buscando fortalecer nuestra cultura, las historias ancestrales, nuestras tradiciones y costumbres, las que tenemos en nuestro pueblo. Entonces a partir de

22- La cursiva es nuestra.

23- Los significados de vida, es una herramienta que propone Green Stócel (2011) para indagar por el origen de las palabras de la lengua Dule y desde allí conocer el alma y el sentido de las cosas que el pueblo Gunadule viene recreando desde su existencia. Este aspecto será profundizado en la última categoría de análisis que se presenta en esta investigación. 
ahí nace este proyecto; para pensar un poco más sobre las matemáticas Dule y continuar investigando. (Fragmento de trabajo de discusión desarrollado para esta investigación del 29 de julio de 2011).

Se pone de manifiesto que una de las tensiones de fondo en lo referido a la escuela y al currículo escolar está relacionada con las formas de organización temporal y espacial de la educación por parte del Estado. Las acciones del maestro, en la escuela del Estado, son muy limitadas para generar relaciones con las escuelas ancestrales ${ }^{24}$. En este sentido, en el convenio MEN-Ibgigundiwala ${ }^{25}$ (2011c) se reconoce que las discusiones sobre el currículo escolar para la comunidad Dule están centradas en medio de tensiones de poder:

Todo lo referente a la educación se debe a un pensamiento político ${ }^{26}$ Dule; desde ahí decidimos y posicionamos qué es lo que queremos como pueblo, nuestro punto de vista. Este proyecto es esencialmente político en la medida que representa una posición sobre la educación nuestra y la que proponen otros pueblos o naciones, tal es el caso de la educación oficial que se imparte en la nación colombiana desde su gobierno central... Se busca complementar el saber propio con saberes y herramientas del saber occidental y de otros saberes que sirvan en nuestro propósito de alimentar el proyecto de la vida... (MEN- Ibgigundiwala, 2011b, p. 4).

\footnotetext{
24- Son las escuelas relativas a las prácticas sociales y elementos culturales que nutren la identidad del pueblo Dule desde la historia ancestral. Estas escuelas son constitutivas de la Casa del Congreso. Algunas de ellas son: escuela de hamacas, escuela de artesanías, escuela de música y danza, escuela de comidas y bebidas, escuelas especializadas (cantos terapéuticos, botánico, Sagla (cacique), nele (chamán), historiador....). Aquí son fundamentales las prácticas sociales para la formación integral en la familia, el Congreso, la Madre Tierra y el Cosmos.

25- Este es un Convenio entre el Estado y el pueblo Dule colombiano enmarcado en la sentencia constitucional T-025 de 2004. En este convenio se desarrolló un trabajo centrado sobre el currículo escolar indígena Dule y la educación del pueblo Dule.

26- La cursiva es nuestra.
}

Estas tensiones se hacen más fuertes cuando se trata de pensar e investigar la producción de conocimiento [matemático] en culturas indígenas. Lo anterior en nuestra investigación ha significado el encuentro con otros sentidos, otras miradas, otras historias en relación con la construcción del conocimiento [matemático] del pueblo Dule.

\section{El maestro indígena Dule en situación de frontera}

En este proceso de investigación emergió como elemento fundamental la experiencia de los profesores indígenas en sus comunidades, pues como Silva (2010) lo plantea, el currículo escolar indígena se convierte en un espacio de producción y creación de significados, pues, en el currículo se producen sentidos e significaos sobre los diferentes campos y actividades sociales; en él se trabaja sobre materiales culturalmente existentes. Así, dice este autor, el currículo es entonces lugar, espacio, territorio, pero ante todo es un espacio de relaciones de poder en el que se pone en juego la constitución de la identidad. En la escuela del Estado, dice Nazario, se tienen unos tiempos y espacios definidos, pues los maestros,

[...] tienen dos gobiernos, el propio y el del Estado. La comunidad lo certifica para que ocupe un cargo y luego lo nombran, pero ahí ya el jefe es otro ${ }^{27}$, el Estado... Pero cada indígena que se va formando, debe pensar en lo suyo, debe llevar su cultura, su identidad y creer en lo suyo, debe relacionarse... El maestro de hoy debe ser estratégico, si la autoridad no da, ellos deben empezar a dar para que la autoridad dé, y las autoridades también deben dar para que los maestros den, si no es así va a ser muy difícil... (Fragmento de entrevista concedida para esta investigación por Nazario Uribe el 27 de julio de 2011).

27- La cursiva es nuestra. 
Al confrontar las diversas situaciones que han emergido en las universidades y en las diferentes comunidades indígenas, respecto a la formación de maestros indígenas, en nuestra investigación fue posible encontrar algunos elementos que nutren las discusiones sobre cómo el maestro indígena Dule, que trabaja en la escuela del Estado y que al mismo tiempo debe seguir las propuestas de su comunidad desde la Casa del Congreso, se encuentra en un punto de frontera entre dos culturas, entre dos espacios. Entendemos por frontera, en el sentido de Silva,

Que esta no es una línea, la frontera es uno de los elementos comunicación social que tiene una función reguladora. Es la expresión de un equilibrio dinámico en el que no solamente se encuentra el sistema territorial... Las fronteras representan mucho más que una mera división $\mathrm{y}$ unificación de dos puntos diversos; ir más allá de las fronteras geográficas, es un campo de diversidad. Es un encuentro con los "diferentes" no solo desde lo físico, sino también desde lo social. Y este espacio es que las relaciones se forman y se deforman; se complementan y dan forma a la diversidad y la cultura. (RAFFESTIN, 2005 apud SILVA, 2010a, p. 213).

En este sentido, las fronteras representan más que una división de un espacio físico; ellas también representan diversos puntos de vista, obstáculos o conflictos en los que los maestros indígenas están incluidos.

Diferente del maestro no indígena, la investidura de maestro indígena no implica ser apenas un profesional y aprender a manejar los intereses de dos puntos de vista para el trabajo en el aula. Ser maestro indígena exige, también y fundamentalmente, estar incluido en un proyecto de vida comunitario. Además, el maestro indígena Dule debe mediar entre dos culturas: en el aula clase, pues la comunidad le asigna la responsabilidad de enseñar a los niños la propia cultura, pero el Estado le pide responder por los contenidos curriculares que fueron establecidos frente desde el modelo educativo de escuela nueva. En este sentido el saila Jaime Melendres hace un llamado a los maestros para afrontar en la escuela la enseñanza dialógica de la cultura occidental y la cultura Dule pues,

Debemos [Los Dule] estudiar ambos conocimientos, si solamente estudiamos el conocimiento del otro significará la muerte de la cultura Dule, prevalecerá la otra cultura, no la de nosotros, por lo tanto ocasionará el aumento y la penetración de la otra cultura en nuestros sistemas de valores y la autoestima de ser Dule. En ese sentido, las generaciones que vienen detrás de nosotros vendrán sin memoria, sin cultura y sometidas a la pobreza. (Fragmento de entrevista concedida para esta investigación por Jaime Melendres el 21 de abril de 2012).

Así, el maestro indígena Dule, incluso dentro de los marcos legales establecidos por el Estado se encuentra como mediador entre las dos culturas. Respecto a la formación que ha sido ofrecida a los maestros indígenas desde el Estado, se ha cuestionado la idea de formar a los maestros indígenas para que traduzcan en la escuela del Estado los conocimientos culturales, provenientes de las prácticas sociales, en los conocimientos occidentales. Estos cuestionamientos están relacionados, como lo expresa Nazario Uribe, con la historia del pueblo Dule en relación con la educación de los niños, niñas y jóvenes,

Hasta donde me contaron mis abuelos, mi papá y mi mamá, prácticamente la educación del pueblo Dule era desde los padres, dese la comunidad, desde los sabios. No había lo que hoy se llama una universidad, un colegio o una escuela; esas son cosas totalmente ajenas al pueblo 
Dule... Entonces, la educación dentro de la formación de pueblo Dule era como ambulatoria... Y para tomar la tarjeta profesional acudian a un sabio en un tiempo determinado definiendo lo que querían aprender... Por ejemplo, botánica, canto, medicina... (Fragmento de entrevista concedida para esta investigación por Nazario Uribe el 29 de julio de 2011).

De ese modo, hay diferencias entre la forma de pensar del pueblo Dule frente a la formación de sus hijos, y el pensamiento y la forma de organización occidental de la escuela y sus currículos. Esto muestra, una vez más, el cuidado que ha de tenerse de parte de investigadores y de instituciones formadoras de maestros, al intentar diálogos interculturales en sus propuestas de formación. Pues, contrario a las creencias de los currículos occidentales, los saberes y prácticas sociales de los pueblos originarios de Abya Yala - pueblos Dule- no son asunto del pasado; esos saberes y prácticas siguen vivos. En palabras de Richard Nixon Cuéllar,

Nosotros los maestros estamos en medio de muchas posiciones... Por ejemplo, los padres de familia dicen: nosotros vamos a decir qué es lo que ustedes van a enseñar, pues ustedes deben hacer lo que nosotros decimos con nuestros hijos. Luego, los padres nos dicen que debemos enseñar solo español, ciencias y sociales. Pero los sabios dicen otras cosas. (Fragmento de trabajo de discusión desarrollado para esta investigación el 26 de julio de 2011).

Este lugar de habitar en una frontera, en la que se encuentran los maestros indígenas Dule, también es una posición de frontera para quienes, de una u otra forma, están pensándose la educación indígena del pueblo Dule -los sabios, la propia comunidad Dule, y algunos investigadores. Para cada uno de ellos siguen existiendo puntos de frontera frente a la escuela del Estado y a la casa del congreso, ya que con el proyecto de un sistema educativo propio "se busca conservar la vida del Pueblo Dule y proteger la Madre Tierra. Esto no lo garantiza sólo el Pueblo Dule: hace parte de un tejido de saberes y acciones con otros pueblos y otros pensamientos" (MEN- Ibigundiguala 2011b, p. 3).

\section{A modo de conclusiones}

Era objetivo de este artículo mostrar algunos de los hallazgos de nuestra investigación basados en dos de las categorías emergentes referidas a elementos que nos posibilitaran ver cómo los maestros Dule han venido (re) significando el currículo escolar indígena relativo al conocimiento [matemático] desde y para las prácticas sociales de la comunidad de Alto Caimán en diálogo intercultural con la escuela del Estado.

En primer lugar, es necesario reconocer el papel que en dicho proceso ha tenido la investigación colaborativa desde el cual asumimos el desarrollo de la investigación, pues los maestros resaltaron la importancia de tener la posibilidad de reunir a personas con diferentes experiencias y diferentes formas de mirar el mismo objeto investigado (el currículo), ya que se posibilitaron marcos interpretativos más amplios. Investigar colaborativamente fue importante para tejer y destejer la problemática y las alternativas concretas frente al proceso de (re)significación del currículo escolar, relativo al conocimiento [matemático], que venía siendo discutido, también desde otros proyectos políticos y educativos, por el pueblo colombiano Dule.

La (re)significación del currículo escolar indígena, relativo al conocimiento [matemático], desde la mirada del maestro indígena Dule, se vio influenciada por la perspectiva crítica del currículo desde la cual asumimos desarrollar esta investigación y los diversos encuentros y actividades. Esta perspectiva, posibilitó que los maestros, más que preocuparse por los contenidos escolares, centraran su mirada en los sujetos que desde la comunidad se 
quiere formar. Lo anterior lo evidenciamos en diferentes momentos desde la voz del maestro indígena Francisco Martínez quien ha venido planteando que se haga efectiva la posibilidad de interacciones interculturales entre la escuela del Estado, la comunidad y los sagalamala.

En este proceso de (re)significación de los maestros, comprendimos que para el currículo escolar indígena -siempre en construcción- es fundamental considerar las prácticas sociales en unidad dialéctica con los conocimientos [matemáticos], de forma indisciplinar. Por eso nuestra apuesta de (re)significación del currículo desde y para las prácticas sociales de la comunidad Dule de Alto Caimán. Por otro lado, identificamos, desde la comunidad, los sailas y desde los maestros, un cuestionamiento hacia los saberes dominantes presentados en la escuela del Estado, pues estos no están asociados a sus prácticas sociales y a los conocimientos [matemáticos] que en ellas son producidos, legitimados y validados. Este es un llamado a reconocer que también la cultura Dule tiene unos conocimientos que le son propios y que constituyen la identidad del Dule.

De este modo, el diálogo con la comunidad Dule nos ha posibilitado el cuestionamiento de lo que hoy llamamos -desde occidente- el conocimiento [matemático]. Para la comunidad Dule, el conocimiento [matemático] no existe, en la lectura que occidente lo comprende. Para la cultura Dule existe el conocimiento. La cultura Dule no disciplinariza, como occidente, el conocimiento. Es por ello que en este trabajo hemos optado por hablar del conocimiento [matemático]. En esta dirección los sailas Jaime Melendres y Faustino Vicente Arteaga nos dijeron,

La palabra matemática no existe en nuestra cultura, existen los valores, contar, medir. Por ejemplo: en la construcción de vivienda, los Dule no dicen que la casa es matemática, sino que ella [la casa] tiene unos sentidos, unos significados, unos conocimientos y unas acciones relacionadas con el hombre [con los
Guna]. Los horcones largos son caciques, los pequeños son voceros; todo completo, la casa, significa la comunidad. Si miramos la casa completa, vamos a ver que todas las partes son cortadas exactamente, es decir, los cálculos son exactos. (Fragmento de trabajo de discusión desarrollado para esta investigación el 20 de abril de 2012).

En este caso, no queremos más referirnos al conocimiento de esta comunidad de forma disciplinarizada; es decir hablaremos de conocimiento y abandonamos la idea de hablar -disciplinaria y fragmentadamente- de conocimiento matemático pues en la cultura Dule no se disciplinariza el conocimiento en la lectura en que occidente lo comprende y lo efectiva mediante la escuela y el currículo. Es por ello que en este trabajo hemos optado por hablar del conocimiento [matemático] entre corchetes, con la finalidad de problematizar el uso de esta palabra para adjetivar o dar sentido a prácticas culturales que son de otra forma de vida que no tiene como referencia las creencias y formas de pensamiento occidentales.

Finalmente, consideramos que el proceso vivido con esta investigación ha posibilitado a los maestros indígenas mirar el currículo escolar indígena Dule como un espacio en el que se exponen y se ratifican las creencias y los conocimientos de su cultura; como un espacio dinámico y constitutivo de la identidad del pueblo Dule. Este espacio de identidad hace más evidente cada uno de los ejes fundamentales de la cosmogonía y la cosmovisión Dule; ejes basados en el respeto a la Madre Tierra. Es desde la Madre Tierra que es posible entender, por ejemplo, y con mayor claridad, los conocimientos [matemáticos] que circulan en las prácticas sociales. En esta propuesta tejida de sueños, pero contundente en la conservación de la identidad Dule, el currículo escolar indígena se convierte en un espacio de producción y creación de significados, pues, como Silva (2009) lo plantea, en el currículo se producen sentidos e significados sobre los 
diferentes campos y actividades sociales; en él se trabaja sobre materiales culturalmente existentes. Así, dice este autor, el currículo es entonces lugar, espacio, territorio, pero ante todo es un espacio de relaciones de poder en el que se pone en juego la constitución de la identidad como una producción social, que nunca está completa, que está siempre en proceso, y es siempre construida en el interior, y no fuera de los procesos de significación, en los que los significados y las prácticas sociales adquieren sentido. Para este autor la identidad está intimamente relacionada con los procesos de producción de la diferencia, por medio de los cuales los grupos culturales de definen y son definidos por otros grupos.

\section{Referencias}

BAJTíN, Mijail. Estética de la creación verbal. México, DF: Siglo XX, 2009.

BAJTíN, Mijail. Problemas de la poética de Dostoievkil. México, DF: Fondo de Cultura Económica, 1986.

BERRÍO, Katherine. La medida en un contexto de escuela indígena: el caso de la comunidad Tule y el caso de la comunidad Embera-Chami. 2009. 91 p. Trabajo de Pregrado -Facultad de Educación, Universidad de Antioquia, Medellín, 2009.

BOAVIDA, Ana Maria; PONTE, João Pedro. Investigación colaborativa: potencialidades y problemas. Traducido por Diego Alejandro Pérez Galeano. Revista Educación y Pedagogía, Medellín, v. 23, n. 59, p. 125-135, ene./abr. 2011.

COLOMBIA. Corte Constitucional: Auto 004/09. Bogotá, [s. n.], 2009. Disponible en: <http://www.dnp.gov.co/LinkClick.aspx?file ticket=yrz6Kcf33mY\%3D\&tabid=1080>. Acceso en: 09 ago. 2011.

COLOMBIA. Ministerio de Educación Nacional (MEN) ¿Qué es la escuela nueva? Bogotá: MEN, 2011a. Disponible en: <http:// www.colombiaaprende.edu.co/html/mediateca/1607/article-89868.html >. Acceso en: 25 mayo 2011.

COLOMBIA. Ministerio de Educación Nacional (MEN) ¿Cómo se aplica el modelo de Escuela Nueva? Bogotá: MEN, 2011b. Disponible en: <http://www.colombiaaprende.edu.co/html/mediateca/1607/article-89868.html>. Acesso en: 16 sept. 2011.

COLOMBIA. Ministerio de Educación Nacional (MEN). Convenio ibgigundiwala. Bogotá: [s. n.], 2011c. Manuscrito sin publicar.

COLOMBIA. Ministerio de Educación Nacional (MEN). Ley general de educación 115. Bogotá, MEN, 1994. Disponible en: <http:// www.unal.edu.co/secretaria/normas/ex/L0115_94.pdf>. Acceso en: 20 mzo. 2010.

D’AMBROSI0, Ubiratan. A etnomatemática no processo desconstrução de uma escola indígena. Em Aberto, Brasília, DF, v. 14, n. 63, p. 93-99, jul./set. 1994. Disponible en: <http://emaberto.inep.gov.br/index.php/emaberto/article/view/949/854>. Acceso en: 12 nov. 2010.

D’AMBROSIO, Ubiratan. Etnomatemática: eslabón entre las tradiciones y la modernidad. México, DF: Limusa, 2008.

D’AMBROSIO, Ubiratan. 0 programa etnomatemática: história, metodologia e pedagogia. 1999. Disponible en: <http://vello sites.uol.com.br/program.htm>. Acceso en: 20 mzo. 2011.

DAVIDOV, Vasili Vasilievich. Problemas do ensino desenvolvimentalista: a experiência da pesquisa teórica e experimental na psicologia. Moscú: Progreso, 1988.

FERRI, Cassia. Currículo multicultural: refletindo a educação escolar indígena. Revista Contrapontos, Itajaí, v. 2, n. 4, p. 82-99, ene./febr. 2002. Disponible en: <http://siaiweb06.univali.br/seer/index.php/rc/article/view/136/116>. Acceso en: 01 febr. 2012.

GREEN STÓCEL, Abadio. Anmal Gaya Burba: significados de vida. 2011. 190 p. Tesis (Doctorado) - Universidad de Antioquia, Medellín, 2011. 
GREEN STÓCEL, Abadio. La lucha de los siete hermanos y su hermana Olowaili en defensa de la madre tierra: hacia la pervivencia cultural del pueblo Kuna Tule. Revista Educación y Pedagogía, Medellín, v. 19, n. 49, p. 227-237, sept/dic. 2007.

GREEN STÓCEL, Abadio; CARDOZO, Marta; OCHOA, Reinaldo. Currículo Tule. Medellín: Organización Indígena de Antioquia (OIA)/ Secretaría de Educación y Cultura de Antioquia, 1995.

HIGUITA, Carolina. La medida desde la medicina tradicional: el caso de una comunidad Embera Chamí. 2011.150 p. Trabajo presentado para optar al título de Licenciada en Educación Básica con Enfásis en Matemáticas - Universidad de Antioquia, Medellín, 2011.

INSTITUTO DEPARTAMENTAL PARA LA EDUCACIÓN INDÍGENA (INDEI). ¿Quiénes somos? Medellín, Indei, 2011. Disponible en: <http://www.indei.org/wordpress/?page_id=2>. Accsso en: 03 jul. 2011.

JARAMILLO, Diana. La educación matemática en una perspectiva sociocultural: tensiones, utopías, futuros posibles. Revista Educación y Pedagogía, Medellín, v. 23, n. 59, p. 13-36, ene./abr. 2011.

KNIJNIK, Gelsa. As novas modalidades de exclusão social: trabalho, conhecimento e educação. Revista Brasileira de Educação, São Paulo, n. 4, p. 35-42, 1997.

MIGUEL, Antonio. Percursos indisciplinares na atividade de pesquisa em história (da educação matemática): entre jogos discursivos como práticas e práticas como jogos discursivos. Bolema, Rio Claro, v. 23, n. 35, p. 1-51, abr. 2010.

MIGUEL, Antonio; MIORIM, Maria. Ângela. Historia en la educación matemática: propuestas y desafíos. Campinas: Autêntica, 2004.

MONTEIRO, Alexandrina. Currículo de matemáticas: reflexões numa perspectiva etnomatemática. In: ENCUENTRO DE EDUCACIÓN MATEMÁTICA, 7., 2005, Tunja. Actas... Tunja: Asociación Colombiana de Educación Matemática, 2005. p. 23-26

MONTEIRO, Alexandrina; MENDES, Jackeline. Prácticas sociales y organización curricular: cuestiones y desafío. Revista Educación y Pedagogía, Medellín, v. 23, n. 59, p. 37-46, ene./abr. 2011.

MONTEIRO, Alexandrina; SENA, Elizabeth; SANTOS, José Augusto. Etnomatemática e prática social: considerações curriculares. In: MENDES, Jackeline; GRAND0, Regina (Org.). Múltiplos olhares: matemática e produção de conhecimento. São Paulo: Musa, 2007. p. 29-63.

MOURA, Manoel. Educar con las matemáticas: saber específico y saber pedagógicos. Revista Educación y Pedagogía, Medellín, v. 23, n. 59, p. 48-57, ene./abr. 2011.

MOURA, Manoel et al. A atividade orientadora de ensino como unidade entre ensino e pprendizagem. In: MOURA, Manoel (Comp.). A atividade pedagógica na teoria histórica-cultural. Brasília, DF: Liber livro, 2010. p. 81-109.

ORGANIZACIÓN INDÍGENA DE ANTIOQUIA (OIA). Información institucional. Medellín, OIA, 2007. Disponible en: <http://www.oia. org.co/pdf/institucional.swf>. Acceso en: 11 nov. 2011.

SÁNCHEZ, Silvio. Fundamentos para la investigación educativa: presupuestos epistemológicos que orientan al investigador. Bogotá: Cooperativa Editorial Magisterio, 1998.

SANTACRUZ, Milton; CASTAÑO, Virginia. Ibisoge Yala Burba Mola ¿Qué nos dicen las Molas de Protección? 2012.160 p. Tesina (Maestría) - Facultad de Educación, Universidad de Antioquia, Medellín, 2012.

SCANDIUZZI, Paulo. Educação indígena x educação escolar indígena: uma relação etnocida em uma pesquisa etnomatemática. São Paulo: Edunesp, 2009.

SILVA, Tomaz Tadeo. Descolonizar el currículo: estrategias para una pedagogía crítica. In: GENTILI, Pablo (Comp.). Cultura, política y currículo: ensayos sobre la crisis de la escuela pública. Buenos Aires: Lozada, 1998. p. 1-9. Disponible en: <http://cem7.edu. ar/index.php?option=com_content\&view=article\&id=168\&ltemid=183 > . Acceso en: 14 sept. 2011.

SILVA, Tomaz Tadeo. Documentos de identidade: uma introdução às teorias do currículo. Belo Horizonte: Autêntica, 2010. 
SILVA, Tomaz Tadeo. El proyecto educacional moderno ¿Identidad terminal? Propuesta Educativa, Buenos Aires, n. 13, p. 0510, dic. 1995. Disponible en: <https://docs.google.com/document/d/1YytgRWdSAsYRubwID_gEL4h_h32ruJecrB2LCWkHMc0/ edit?hl=en_US\&pli=1>. Acceso en: 10 jun. 2011.

SILVA, Tomaz Tadeo. 0 currículo como fetiche: a poética e a política do texto curricular. Belo Horizonte: Autêntica, 1999.

SILVA, Zenete Ruiz. Educação e intercultura para além da fronteira. Revista Espaço Pedagógico, Passo Fundo, v. 17, n. 2, p. 211-222, jul./dic. 2010a.

TAMAYO-OSORIO, Carolina. (Re)significación del currículo escolar indígena, relativo al conocimiento [matemático], desde y para las prácticas sociales: el caso de los maestros indígenas Dule de la comunidad de Alto Caimán. 2012. 160 p. (Tesis de Maestría en Educación) - Facultad de Educación. Universidad de Antioquia, Medellín, 2012.

WALSH, Catherine. Interculturalidad, plurinacionalidad y decolonialidad: las insurgencias político-epistémicas de refundar el Estado. Tabula Rasa, Bogotá, n. 9, p. 131-152, 2008.

Recibido en 09.02.2015

Aprobado en 17.12.2015

Carolina Tamayo-Osorio es alumna de doctorado en la Facultad de Educación de la Universidade de Campinas (Unicamp). Posee maestría en educación en la Universidad de Antioquia (2012). Es integrante de los grupos de investigación PHALAEducação, Linguajem e Práticas Culturais de la Facultad de Educación de la Unicamp y Matemática, Educación y SociedadMES, de la Universida de Antioquia. 